\title{
Cystoid Macular Edema: Causes, Diagnosis and Treatment
}

Noelia Bravo-Alcobendas, ' Joseba Zulueta, ${ }^{1}$ Elena Salobrar-García, Juan J Salazar, ${ }^{1}$ José M Ramírez.

\begin{abstract}
The purpose of this paper is to conduct a review of studies on cystoid macular edema published in the last seven years. Cystoid macular edema is a major cause of loss of visual acuity. It is the final common pathway of many diseases and can be caused by numerous processes including inflammatory, vascular, adverse drug reactions, retinal dystrophy or intraocular tumors. These processes disrupt the blood-retinal barrier, with fluid extravasation to the macular parenchyma. Imaging tests are essential for both detection and monitoring of this pathology. Fluorescein angiography and autofluorescence show the leakage of liquid from perifoveal vessels into the tissue where it forms cystic spaces. Optical coherence tomography is currently the gold standard technique for diagnosis and monitoring. This allows objective measurement of retinal thickness, which correlates with visual acuity and provides more complete morphological information. Based on the underlying etiology, the therapeutic approach can be either surgical or medical with anti-inflammatory drugs. We found that disruption of the blood-retinal barrier for various reasons is the key point in the pathogenesis of cystoid macular edema, therefore we believe that studies on its treatment should proceed on this path.
\end{abstract}

Keywords: Macular edema, Etiology, diagnosis, ophthalmology, therapeutics (Source: MeSH, NLM).

About the author: Noelia Bravo is currently a 6 th year medical student of Universidad Complutense, Madrid, Spain of a 6 year program.

Joseba Zulueta is currently a 6 th year medical student of Universidad Compluten. se, Madrid, Spain of a 6 year program.

\section{Introduction}

Cystoid macular edema (CME) is the accumulation of fluid in the retina between the outer plexiform layer and the inner nuclear layer around the fovea, which results in the formation of cysts. In the long term, these cysts may coalesce into large cystic spaces, irreversibly damaging the central vision. This review presents a brief synopsis of the pathophysiology, diagnosis, management and treatment of CME. In addition, many publications contain information on the CME but not addressed globally, and that is what this article is intended to achieve. The aim of this publication is to present a review of studies published on CME in recent years, to provide a more current view as the management of this constantly evolving condition.

\section{Search Strategy and Selection Criteria}

A literature search was performed up to July 2014 using the MEDLINE database, PubMed, and Google Scholar search services with the following key words and word combinations: cystoid macular edema, macular edema, CME treatment, CME diagnosis, CME etiology, Irvine-Gass syndrome.

A total of 3728 articles were found. After filtering by author criteria (articles published between 2008 and July 2014), English or Spanish language, and the condition that they all addressed cystoid macular edema as the main theme (pathophysiology, diagnosis and treatment) and not merely as a complication of another disease), 26 articles were included after a full text review. All the abstracts were then carefully divided into subcategories covering topics including pathogenesis, etiology, clinical manifestations, research and treatment of macular edema.
This review covers systematic reviews, original articles, a letter to the editor and an interpretive essay (Figure 1). We did not contact other authors for further articles inclusion.

Figure 1. Flow Diagram Showing the Number of Studies Included in and Excluded From the Review.

References identified in

databases by computeri-

zed search $(n=3728)$

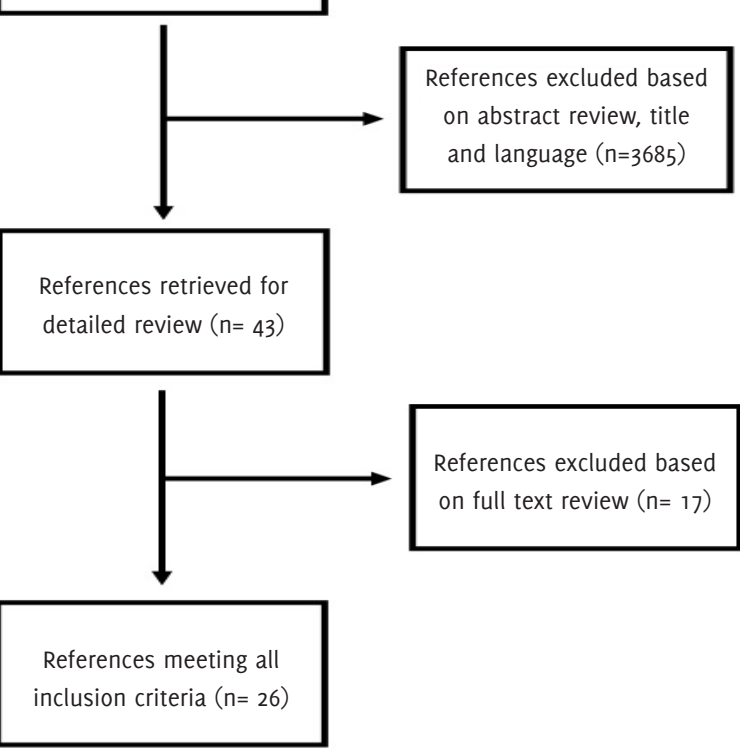




\section{Pathophysiology of CME}

CME is a common form of response of the retina to different insults:

\section{Postsurgical CME}

Also known as the Irvine-Gass syndrome, ${ }^{1}$ this is a major cause of clinically significant reduced vision after intraocular surgery which can occur even in the absence of complications during surgery (about $1 \%$ of post-surgical CME). ${ }^{2-5}$ The incidence is higher (up to 20\%) ${ }^{6}$ after cataract surgery, although it can occur after penetrating keratoplasty, glaucoma surgery or YAC laser capsulotomy following panretinal photocoagulation. However, it has been observed that the use of phacoemulsification significantly reduces postoperative CME (0.1-2.35\% with this technique), and in cases where it does develop, it is often limited. CME may be acute (when it appears during the first 4 months after surgery), late (if it appears later than 4 months), chronic (if it happens after 6 months) or recurrent. ${ }^{3}$ It usually appears between 4 and 12 weeks after cataract surgery, although it can occur years later. ${ }^{7,8}$

The pathogenesis of CME is under discussion, although some theories posit the existence of inflammatory factors -prostaglandins (PG), leucotriens (LT)- that increase vascular permeability causing fluid accumulation in cysts, or changes in protein composition of the vitreous humour, as a result of the surgical trauma. ${ }^{2,3,8,9}$ Also, vitreomacular traction, ${ }^{3,8,9}$ vitreouveal traction, ${ }^{3}$ vitreous incarceration, ${ }^{3}$ loss of vitreous humour, ${ }^{,}$posterior capsule rupture ${ }^{8}$ or hypotonic vitreous ${ }^{8,9}$ may contribute to the development of a CME and likewise iatrogenic iris injuries, retinal vein occlusions or even the presence of epiretinal membranes. However, it has been observed that posterior vitreous detachment could be a protective factor. ${ }^{10}$

The incidence of CME after cataract surgery varies depending on the type of procedure, the surgeon's experience and the patient's profile (comorbidities such as systemic diseases and other eye disorders). ${ }^{8}$ The most important risk factors associated with this process include Diabetes Mellitus (DM), ,.9 hypertension (HT), ${ }^{5}$ and old age, ${ }^{9}$ as well as preexisting conditions such as uveitis, ${ }^{7}$ retinal vein occlusion, ${ }^{5}$ and the presence of epiretinal membranes. ${ }^{3}$

\section{Uveitis}

CME is the most common complication of uveitis and the most common cause of visual loss in patients with intraocular inflammatory diseases. ${ }^{5}$ Compared to Irvine-Gass Syndrome, uveitic CME seems to have a worse prognosis and greater impact on the visual acuity (VA) of affected patients, especially older patients or those with concomitant chronic diseases. ${ }^{2}$ It typically occurs in patients with an intermediate or posterior uveitic component but can also be found in patients who only suffer from HLA-B27-Associated acute anterior uveitis., ${ }^{2,5}$, It usually appears in patients with autoimmune uveitis (pars planitis, birdshot retinochoroidopathy, Vogt-Koyanagi-Harada syndrome, Behçet's disease) 5 or infectious (toxoplasmosis), 2,5,7 toxic (associated with rifabutin) ${ }^{2}$ or idiopathic (sarcoidosis, idiopathic vitritis) $)^{2}$ disorders.

The pathogenesis is not well defined, but it is known that the most important mechanism is the loss of integrity of the BRB, caused by inflammatory mediators generated by uveitis (including PG, LT, interleukins, tumour necrosis factor alpha, vascular endothelial growth factor [VEGF]). ${ }^{2,5,7}$ If edema is caused by pars-planitis, it disappears within 6-9 months, and it is possible to restore vision; however, if it persists beyond this time, chronic macular changes occur and the CME will cause permanent vision damage. ${ }^{5.7}$ Some authors, therefore, recommend intensive management, since this achieves long-term visual improvement in most patients. In fact, it has been found that $V A$ in the first month of follow-up after treatment is similar to VA throughout the follow-up period. ${ }^{11}$

There are two factors that may be associated with a better outcome in the long term: good VA at the beginning of the treatment (it has been seen that the photoreceptors are capable of recovering a fraction of visual function with treatment, so that patients with less damage at the beginning will recover their VA better); and lower age (this association with age is independent of the duration of uveitis or duration of the follow-up). ${ }^{11}$

\section{Laser}

CME development or the worsening of an existing CME is a complication of panretinal photocoagulation used in the treatment of vascular retinopathy, or after YAG laser posterior capsulotomy. ${ }^{5}$ It usually occurs between 1 and 5 months after the procedure and has been observed to relate more to the opening of the posterior capsule than to laser energy. ${ }^{2}$ It is suspected that laser induces the production of inflammatory mediators and would also enhance fluid extravasation by increasing the vascular flow to the macula. ${ }^{2,5}$

\section{Retinal vein occlusion}

The occlusion of both the central retinal vein and its branches is a common cause of CME. ${ }^{5,7,12}$ The pathogenesis of this disease is not clearly understood. Some authors support the idea that thrombotic occlusion of the vein is responsible for the clinical manifestations of CME in this case; others claim that the cause is inflammation, but we cannot rule out the possibility that both act in a complementary manner. ${ }^{5}$

In the first case, the vein occlusion causes a rise in intravascular pressure in retinal veins distal to the occlusion site. By increasing the hydrostatic pressure, it causes transudation of fluid into the extravascular space. ${ }^{5,13}$ In the second case, on the other hand, BRB rupture mediated by cytokines expressed in hypoxic retina (VEGF, IL6 or factors derived from the retinal pigment epithelium [RPE]) may lead to fluid extravasation. ${ }^{5}$

There are a number of concurrent processes that can worsen vision loss, such as macular haemorrhage2, macular ischaemia, ${ }^{2}$ submacular fluid with secondary damage to the RPE, ${ }^{2}$ traction by epiretinal membranes ${ }^{13}$ or vitreomacular attachments. ${ }^{5}$ The development of fluid blood levels in the retina is common following obstructive venous retinopathy, although this can also be found in many other retinal diseases. ${ }^{5.7}$ CME resulting from either central or peripheral occlusion tends to be chronic (> 8 months) and difficult to manage. ${ }^{5,7}$ Persistent CME may be associated with hyperlipidaemia, cardiovascular history or vitreomacular adhesions and correlates inversely with glaucoma.? 


\section{Diabetic retinopathy}

Diabetic CME is the leading cause of vision loss in diabetic patients. ${ }^{57,12,14}$ The pathogenesis is related to an increase in retinal blood flow resulting in extravasation of fluid into the parenchyma, but BRB rupture may be more important. ${ }^{5,13}$ The latter seems to be triggered by chronic hyperglycaemia typically affecting the vessels of these patients, which in turn triggers a series of metabolic events that culminate with increased expression of inflammatory cytokines such as VEGF, which can change vascular permeability. This whole process is enhanced by the presence of partial vitreomacular adhesions caused by partial vitreous detachment. ${ }^{5}$

\section{Radiation}

CME is a common manifestation in patients who have received radiation in the head and neck areas. ${ }^{15}$ The retinopathy signs are similar to those in diabetic retinopathy, including CME, which is the main cause of vision loss in radiation retinopathy. ${ }^{5}$ Its incidence depends on the total dose and daily fraction, but CME changes often develop from 30-35 Gray (Gy), usually between 6 months and 3 years from the beginning of the treatment. Bilateral retinopathy occurs in at least half of patients treated with external beam radiation. ${ }^{15}$

\section{Drug reactions}

CME can be caused by drugs such as adrenaline, nicotinic acid and topical latanoprost (prostaglandin analogue). Topical $2 \%$ adrenaline may produce cystic changes by reducing blood flow to the retina and choroid. The nicotinic acid used for the treatment of hypercholesterolaemia leads to the formation of cystic spaces in the inner nuclear layer and outer plexiform layer, which are solved by discontinuing the drug. ${ }^{.}$It is thought to damage the BRB and cause CME, which is angiographically detectable during the early postoperative period in pseudophakic patients. $^{15}$

A case of CME secondary to the use of risperidone has been published recently. Its authors believe CME is dose-dependent, as it disappears after drug withdrawal. The causative mechanism appears to be related to vasodilation of retinal vessels secondary to adrenergic blockade exerted by the drug, although this effect has not yet been confirmed in humans. ${ }^{16}$ Other drugs associated with CME are docetaxel, ${ }^{17}$ paclitaxel, ${ }^{18}$ tamoxifen, ${ }^{5}$ and glitazones. ${ }^{19}$

\section{Retinal dystrophies}

CME occurs in $10 \%$ of patients with retinitis pigmentosa. 5 The perifoveal capillaries show increased permeability or reversed polarity in degenerating RPE cells. ${ }^{15}$ It is believed that antibodies may have a role in the pathophysiology of CME. ${ }^{5,7}$

\section{Traction maculopathies}

Vitreous traction syndrome is characterized by partial separation of the peripheral vitreous with persistent adherences to the macula. ${ }^{5}$ The syndrome may include foveal cysts (considered a prerequisite to the development of a macular hole), epiretinal membranes, macular holes and total lamellar thickness and tractional CME. The natural history of the syndro$\mathrm{me}$, its prognosis and treatment depend on the size of the vitreomacular residual adhesions and consequent macular anatomical changes. ${ }^{20}$

\section{Intraocular tumours}

CME is associated with tumours such as choroidal nevi, malignant melanomas, retinal capillary haemangioma or cavernous haemangiomas. ${ }^{5}$ Cystoid changes occur on the tumour and at sites distant from it because of the lack of retinal oxygenation. ${ }^{15}$

\section{Diagnosis}

CME is associated with two fundamental processes, the accumulation of abnormal extracellular fluid and the formation of cystic spaces. There are various diagnostic tests to confirm these findings. Some, like fundus fluorescein angiography (FA) or autofluorescence imaging, are geared to detect abnormalities in the BRB.? Others, like optical coherence tomography (OCT), are aimed at detecting retinal thickening. ${ }^{7,14}$ The detection of CME in these tests does not always mean it is clinically significant, as clinical CME is defined as a decrease in vision to $20 / 40$ or less that is detectable by FA or $\mathrm{OCT}^{2}$ The most common symptoms and signs in CME are blurring or central vision loss, as well as edema and painless swelling of the retina. Most cases are asymptomatic and are only detected by the imaging techniques mentioned. ${ }^{2}$

\section{1. $O C T$}

OCT is based on low-coherence interferometry, typically using near-infrared light. Relatively long-wavelength light can be used to penetrate the scattering medium and thus obtain high-resolution cross sections of the sensory retina, providing morphological information on the CME. ${ }^{12}$ This allows the physician to make accurate retinal ${ }^{13,21}$ and choroid thickness measurements. ${ }^{7,13}$

Measuring retinal thickness is crucial since there is a negative correlation between thickness in the centre of the macula and VA. ${ }^{3,14}$ This is because the neuroretina requires the bipolar cells to be intact in order to maintain a connection between the photoreceptors and ganglion cells, so that when the accumulation of fluid in cysts exceeds the elasticity of bipolar cells, the axons break up and the neural transmission is interrupted. ${ }^{14}$ Some authors assert that when the cysts fuse and separate the inner and outer layers of the retina, the loss of neural transmission may not lead to any improvement of VA, even when CME is resolved, which is also associated with atrophic changes and thinning of the macula, both observable with $0 \mathrm{CT} .^{22}$

Choroidal thickness is increasingly being seen as important in some pathologies causing CME, as in the case of DM, where some authors have reported increased thickness of the choroid directly proportional to the degree of diabetic retinopathy. ${ }^{23}$

OCT is an objective test that is highly sensitive regardless of the etiology of the CME ( $95 \%$ sensitivity for detection of definite CME, when compared to fluorescein angiography (44\%), in series where questionable grades were interpreted as nonCME cases; $83 \%$ sensitivity when compared to $\mathrm{AFG}(74 \%)$ in series where questionable grades were interpreted as positive CME cases). ${ }^{12}$ The great advantage of this technique is that it is fast, non-invasive and does not cause discomfort to the patient. ${ }^{12,21}$ OCT is considered the current gold standard for CME diagnosis and is also used for monitoring treatment (Figure 2) $3,7,12,14,21$ 
Figure 2. OCT Scans of the Macula. (A) Cystoid macular edema secondary to diabetes mellitus type-II, showing the presence of cysts within the inner nuclear layer. The absence of the normal foveal depression can also be observed. (B) Morphological recovery after photocoagulation treatment and systemic control of the patient's diabetes. (C,D) Three-dimensional reconstruction of the fovea before (C) and after (D) the treatment.
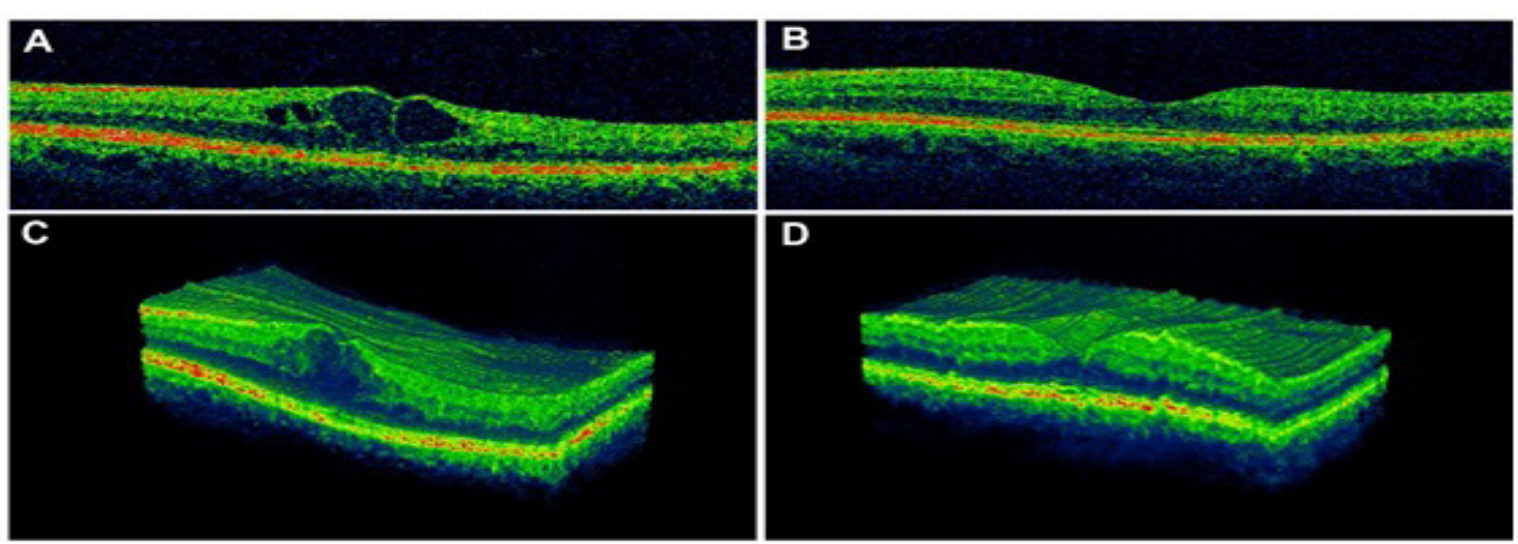

Lengend: Original images from the image bank of the "Instituto de Investigaciones Oftalmológicas Ramón Castroviejo (UCM)".

Figure 3. Macular Hyperfluorescence Observed with Fluorescein Angiography in a Diabetic Patient. Petaloid pattern secondary to contrast leakage from perifoveal capillaries to the cystic spaces. There are also panphotocoagulation scars all around the retina.

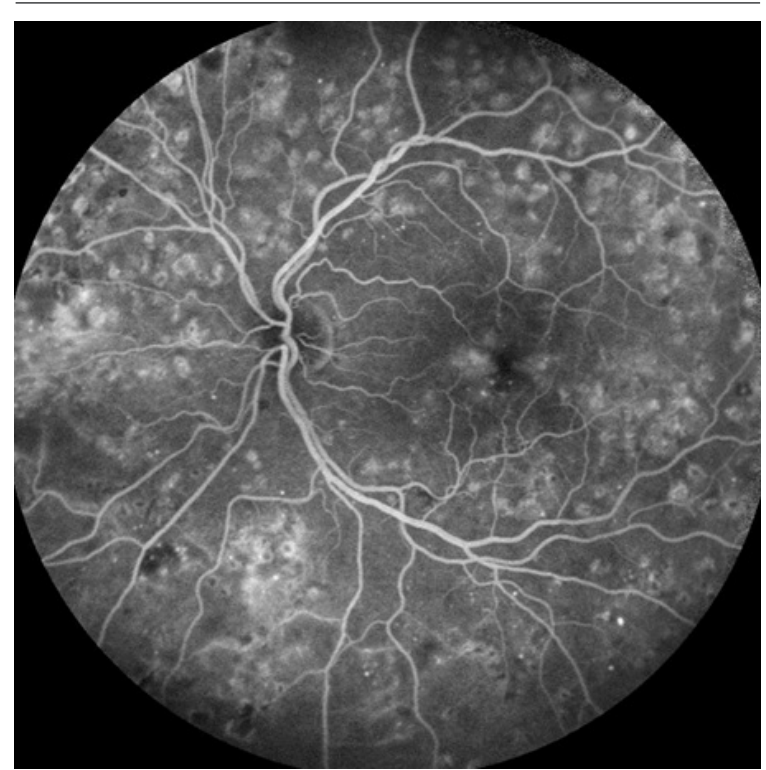

Lengend: Original images from the image bank of the "Instituto de Investigaciones Oftalmológicas Ramón Castroviejo (UCM)".

\section{Fluorescein angiography (FA)}

When FA is used to examine the fundus, the circulation in the retina and choroid can be studied by injecting a contrast that shines when stimulated with light in the blue wavelength range between 265 and $490 \mathrm{~nm}$ (fluorescein). This provides functional and qualitative information that enables the physician to locate and describe the pattern of vascular leakage. FA shows the extravasation of fluid from the perifoveal capillaries in the early stages and the accumulation of contrast in a petaloid, honeycomb or diffuse pattern in later stages., ${ }^{3,12,24}$ However, it has the disadvantage of being invasive and its interpretation depends on the physician (Figure 3). ${ }^{12}$

Contrast extravasation does not always imply an accumulation of intraretinal fluid, as there may be situations where the liquid does not accumulate despite the contrast because it is escaping from the vessels at the same rate as it is being pumped in by the RPE. The opposite situation is also possible; for example, there could be an accumulation of fluid without hyperfluorescence. This happens when the escape point is very small, so that the molecules escape slowly and disperse quickly in the cystic space and are thus not detected by the FA in the standard period of time (10 minutes). ${ }^{12}$

Diagnosis can also be difficult in cases where it is not possible to discern whether the fluid observed belongs to cysts in the retina or other pathological conditions of the retina where contrast extravasation occurs (such as choroidal neovascularization or RPE alteration). ${ }^{12}$ This test is used for clinical diagnosis of CME as well as to perform the treatment. A relationship has been observed between loss of VA and the presence of cysts, although there is no proven relationship between VA and the distance of the cysts from the fovea. $3,7,12,13$

\section{Autofluorescence}

Autofluorescence occurs through the absorption of short wavelength light by the lipofuscin from the RPE cells and emission at a longer light wavelength. This is due to the presence of lipofuscin (an autofluorescent substance that increases with age, cell stress and oxidative damage) in the lysosomes of such cells. When RPE is not viable autofluorescence from those cells disappears. Normally RPE autofluorescence is masked by the pigments in the outer plexiform membrane; however, this does not happen in CME, where the fluid accumulating in the cyst redistributes Henle fibres in that layer, allowing us to observe the natural fluorescence of the RPE. ${ }^{24}$ This technique can be used to monitor patients with CME and could be an alternative for patients who have a history of adverse reactions when fluorescein is injected ( $81 \%$ sensitivity, $69 \%$ specificity). ${ }^{24} \mathrm{Also}$, it is a minimally invasive test and is quick to perform. ${ }^{7,24}$

However, this technique has some disadvantages: firstly, the lens (which opacifies with age) interferes with the fluorescence of the RPE because it is excited at a wavelength similar to the one on which this technique operates, so that high contrast images cannot be obtained. Secondly, it is not considered an accurate way to assess the severity of CME, although it can be 
used as an alternative when it is not possible to perform the OCT. Another limitation is the presence of concomitant pathology of retinal layers and the RPE, because it interferes with the autofluorescence pattern. Finally, this test is not useful if the patient has a non-cystic macular edema because these scatter less pigment. ${ }^{24}$

\section{Management and Prevention 1. Anti-nflammatory drugs (NSAIDS)}

In aphakic or pseudophakic CME, the occurrence of intraocular inflammation with synthesis of prostaglandins results in disruption of the tight junctions of the perifoveal retinal capillaries.' capillaries. NSAIDs inhibit the conversion of arachidonic acid to endoperoxides and thus inhibit the synthesis of $P G$, which reduces fluid leakage from capillaries. ${ }^{2,7}$ In addition, some NSAIDs, such as diclofenac, act on other mediators to inhibit the formation of lipoxygenase products, which are required for the production of the $\mathrm{PG}{ }^{25}$

NSAIDs are currently available in different formulations and administration routes, such as oral, topical or intravitreal. Increased ocular penetration of NSAIDs has been observed when they are administered topically rather than systemically. 3 The most widely used topical NSAIDs are $0.5 \%$ ketorolac, $1 \%$ indomethacin and $1 \%$ diclofenac, which are useful for treatment and prevention of postsurgical CME. ${ }^{26,27}$ In fact, there are studies suggesting that NSAIDs are more effective than corticosteroids in preventing postoperative $\mathrm{CME},{ }^{910}$ especially if administered between 1 and 3 days before surgery. 10 They are also useful for prevention of angiographic edema, although the decrease in retinal thickness and edema does not always correlate with an improvement in VA. ${ }^{3,28}$

The main complications of this treatment include, in descending order of frequency, irritation, burning, stinging, ocular discomfort, conjunctival injection, punctate keratitis, corneal melting, mydriasis, delayed wound healing, hypersensitivity and allergic reactions. ${ }^{9.10}$ When combined with intravitreal steroid therapies or with anti-VEGF injections, these drugs can enhance the effect of the former in chronic pseudophakic CME. ${ }^{29}$

\section{Glucocorticoids}

Glucocorticoids inhibit the production of PG and LT by inhibiting the phospholipase A2 enzyme. ${ }^{2,30}$ Besides their vasoconstrictor properties, steroids reduce intracellular and extracellular edema and hijack $T$ lymphocytes from circulation, inhibiting recruitment and cytotoxic activity. They also suppress the activity of macrophages and polymorphonuclear cells and reduce lymphokine production.?

In the case of post-surgical CME, no difference has been found in the control of inflammation when comparing the power of the corticosteroids employed. It has not been proven that steroids increase bleeding time after eye surgery, but it is something to be aware of. ${ }^{10}$

Corticosteroids can be administered by different routes:

- Topical steroids: prednisolone acetate lipophilic suspensions easily penetrate through the intact corneal epithelium and reach the anterior chamber, achieving higher concentrations than hydrosoluble suspensions such as dexamethasone. ${ }^{31}$
The power in descending order is: $0.1 \%$ dexamethasone, $1 \%$ prednisolone, $0.1 \%$ fluorometholone, $1 \%$ rimexolone, $0.5 \%$ loteprednol and $1 \%$ medrysone. These properties are useful in the treatment of CME caused by chronic iridocyclitis or iritis.? ocular complications of topical steroids include an increase in intraocular pressure (IOP), posterior subcapsular cataract, increased incidence of ocular viral infections, mydriasis, ptosis and eyelid skin atrophy. ${ }^{15}$

- Periocular sub-Tenon injection: penetrates through the sclera generating a maximum and lasting response. This route of administration allows physicians to use water soluble steroids. One of the possible risks is eyeball penetration. In addition, it is contraindicated in certain groups of patients such as steroid-induced glaucoma, hypersensitivity to any of its components, necrotizing scleritis and active ocular toxoplasmosis. ${ }^{15}$

- Intravitreal Injection: triamcinolone acetonide is the most commonly used drug. It is especially used in difficult-to-treat cases such as refractory pseudophakic CME, ${ }^{3}$ uveitic CME and CME secondary to venous occlusion. ${ }^{29}$ It has the same risks and contraindications as the foregoing routes; it also includes increased risk of developing glaucoma ${ }^{8}$ and the possibility of retinal detachment, ${ }^{5,7}$ endophthalmitis, ${ }^{2,5}$ lenticular trauma and recurrence after discontinuation of injections. ${ }^{5}$ Furthermore, it has been observed that most patients do not show sustained improvement over time despite receiving multiple injections. ${ }^{2,5}$ Intravitreal implants are currently being developed; these release the drug at a steady rate and could be an alternative in patients who are poorly controlled or are intolerant of repeated injections, systemic steroids or immunosuppressive agents. . $32,33^{2}$ It has been shown that they are not toxic and they maintain constant intraocular levels of the drug for a long period of time (3-6 months, peaking within the first week). ${ }^{34}$ For instance, intraocular implants help reduce uveitic recurrences, reduce the need for adjuvant therapy and improve VA through the reduction of retinal thickness, as has been demonstrated by OCT studies. ${ }^{35}$ However, there can be side effects, such as increased $10 \mathrm{P}^{36}$ cataract, ${ }^{37,38}$ retinal detachment, ${ }^{39}$ and endophthalmitis. ${ }^{37,38}$ The other drawback of these devices is that they must be surgically removed once they cease to exert their effect. Researchers are therefore developing new biodegradable devices that gradually dissolve into the vitreous, avoiding the risks entailed in surgical removal. ${ }^{40,41}$

- Systemic steroids: especially indicated in CME produced by pars planitis or birdshot chorioretinopathy. Short-term effects can include peptic ulcer, aseptic necrosis of the femoral head and disturbances such as euphoria, insomnia and psychosis. Possible long-term adverse effects include osteoporosis, Cushingoid state, tuberculosis, myopathy, suppression of pituitary-adrenal axis and the worsening of preexisting HT or DM. It is therefore very important to monitor the patient, especially in the first two weeks. Systemic steroids are most commonly administered orally or parenterally. ${ }^{15}$

\section{Immunosuppressive agents}

In cases of severe uveitis requiring long-term treatment with medium to high doses of corticosteroids, the use of immunosuppressive drugs should be considered, since studies show that intravitreal methotrexate can reduce the CME and improve VA. ${ }^{2,42}$ 


\section{Carbonic anhydrase inhibitor: Acetazolamide}

Carbonic anhydrase inhibitors increase the transport of fluid through the RPE from the subretinal space to the choroid, reducing edema. ${ }^{3,43}$ They seem to be especially useful in the treatment of CME secondary to retinitis pigmentosa, although these drugs have been used in studies of postoperative CME. ${ }^{3,8,44}$ Eighty per cent of patients treated with acetazolamide have demonstrated VA improvement; however, the therapeutic effect appears to be independent of the reduction of macular edema. ${ }^{44}$

Usually, an initial dose of $500 \mathrm{mg} /$ day is administered for at least a month, and then the dose will be modified depending on the response. ${ }^{7}$ The most common side effects include numbness, fatigue, depression and loss of libido. ${ }^{3}$

\section{Anti-VEGF}

Anti-VEGF drugs block the effects of this inflammatory factor thereby inhibiting its function (endothelial joint relaxant) and reducing vascular permeability and leakage of fluid into the macula. ${ }^{45}$ This group of drugs includes pegaptanib (selective), ranibizumab and bevacizumab (nonselective). While ranibizumab has been proven to be safe and beneficial for VA improvement by reducing retinal thickness in patients with pseudophakic CME, ${ }^{5,46}$ bevacizumab has not yet been approved for ophthalmic use. In fact, it is still being studied for the treatment of CME of inflammatory origin (especially secondary to retinal central vein occlusion, uveitis or after cataract surgery). ${ }^{47-50}$

These intravitreal anti-VEGFs are promising for the treatment of refractory CME; however, the Food and Drug Administration (FDA) has not yet approved them for this pathology. Studies on diabetic macular edema show benefits in VA when using anti-VEGFs. However, a clear improvement has not been seen in VA and macular thickness in uveitic CME, where it is believed that these drugs have a transient, modest and variable stabilizing effect. ${ }^{5,47}$ As in the case of injected steroids, there is a high recurrence rate when the treatment is discontinued, so it also requires repeated injections (which may lead to endophthalmitis). ${ }^{28}$ Therefore, studies are currently in progress on new routes of administration. However, unlike steroids, anti-VEGFs have the advantage of not causing cataract or increasing 10P. ${ }^{47}$ For all the above reasons, some authors recommend reserving these drugs for cases that are refractory to conventional treatments. ${ }^{48}$

\section{Interferon}

Interferon plays an important role in the treatment of autoimmune diseases (such as Behçet's disease) and appears to be useful for reducing intraocular inflammation. Its side effects include 'flu-like' symptoms, fatigue, hepatotoxicity and psychological disorders. ${ }^{2}$

\section{Somatostatin analogues: octreotide}

It has been observed that these drugs reduce edema in $70 \%$ of persistent uveitic CME cases. This response seems to be related to the duration of the CME prior to starting treatment. ${ }^{2}$

\section{Hyperbaric oxygen therapy}

Hyperbaric oxygen has been proposed by some authors as therapy for $\mathrm{CME}$ due to its potential vasoconstrictor effect, which may reduce venous pressure, allowing stabilization of the tight junctions of endothelial cells. There are no conclusive studies regarding the effectiveness of this therapy. ${ }^{29}$

\section{Photocoagulation}

This technique employs a powerful light source to coagulate tissue. Several theories have been put forward to explain the beneficial effect of laser in patients with CME. On the one hand, it appears that cells respond to damage by creating new connections in a few weeks, which restores the integrity of the RPE. ${ }^{39}$ On the other hand, an alternative hypothesis suggests that laser destroys photoreceptors, thereby reducing the consumption of oxygen in the outer retina. This allows only a little oxygen to diffuse from the choroid to the inner retina and thus limits hypoxia. All these changes promote vasoconstriction, which leads to a reduction of hydrostatic pressure, further reducing the pressure and the diameter of the vessels and hence reducing the fluid outflow from the veins. Diabetic macular edema and macular edema following occlusion of a retinal vein branch thus seem to improve after laser application. However, this treatment is not indicated when there is predominance of ischaemic maculopathy. ${ }^{51}$

Panretinal photocoagulation is usually performed after or simultaneously with anti-inflammatory steroid treatment, because inflammation generated by the laser alters the flow and thereby the retinal macular function, reducing VA. ${ }^{52,53}$

Focal laser treatment is used to obliterate microaneurysms that cause focal fluid leakage. Grid photocoagulation may reduce leakage attributable to abnormalities of dilated macular capillaries, with a positive effect on VA and fluorescein extravasation in diabetic $\mathrm{CME}^{7}$ radiation retinopathy, ${ }^{5}$ and $\mathrm{CME}$ secondary to occlusion of a retinal vein branch.7 The main collateral damage associated with this treatment is the formation of scotoma, usually in laser-burnt areas, which disappear within a few weeks. ${ }^{52}$

\section{Vitrectomy}

This technique is reserved for refractory cases. ${ }^{3}$ Its usefulness in CME lies in its effect of removing vitreoretinal adhesions, 5,54 and inflammatory mediators present in the vitreous humour, 5,54 plus it allows better steroid access to the posterior pole. It is useful for CME with vitreo-macular tractions in pseudophakic, phakic, or diabetic patients (who have a higher incidence of vitreous detachment compared with non-diabetic patients). ${ }^{55}$ It is crucial to eliminate the adhesions to resolve this $\mathrm{CME}$, and so it is necessary to complete the vitreous detachment; this can occur spontaneously or may be achieved by surgery (vitrectomy), although recent studies are reporting favourable results with pharmacological vitreolysis. ${ }^{20}$

Compared with laser treatment, vitrectomy appears to improve VA significantly, especially combined with removal of the posterior cortical membrane. ${ }^{5}$ Some studies have concluded that this technique achieves no better results than intravitreal triamcinolone administration in pseudophakic patients in the long term. ${ }^{8}$ Despite its effectiveness, vitrectomy can lead to complications such as cataracts, retinal detachment, vitreous haemorrhage and recurrent glaucoma secondary to an increase in IOP.? 


\section{Discussion}

When we pooled our findings, we found that CME is a common cause of loss of VA for many diseases, regardless of the cause. So even if it is a single entity, authors approaching CME from different points of view are not agreed on the pathophysiology, although they all point in similar directions, such as fluid extravasation to the retina due to loss of BRB integrity or increased intravascular pressure aided by inflammatory mechanisms. However, there must be factors that are not yet known since some cases heal spontaneously. ${ }^{3}$

An understanding of the pathophysiology is essential for effective treatment, but it is just as important to know the right moment to begin the treatment. Avoiding treatment delay is crucial to prevent permanent loss of VA associated with irreversible tissue damage.

The use of nonsteroidal and steroidal agents appears to reduce the incidence of postoperative $\mathrm{CME}^{56}$ while a relationship has been detected between the use of latanoprost after surgery and greater disruption of the blood-retinal barrier (BRB); $3,5,7$ although there are studies suggesting that in most patients the CME may resolve spontaneously without any treatment after surgery. ${ }^{8}$

Some authors report that there is little more improvement of VA after the first month of treatment. ${ }^{9}$ Chronicity of CME impe- des recovery of VA, which leads us to believe that treatment should be aggressive especially during the first month. It is necessary to establish precisely the time and the circumstances in which to start treatment, since CME does not develop immediately, but after a period of latency.

For purposes of diagnosis and further research, the consensus seems to be that for the present $\mathrm{OCT}$ is the best test, given the following advantages: it is non-invasive, it is more objective than other tests, and results are less affected by comorbidities.

\section{Conclusion}

In conclusion, fluid extravasation to the retina due to loss of BRB integrity or increased intravascular pressure aided by inflammatory mechanisms could be the generic factors that take place in the diverse pathologies that can lead to a CME. It is essential to identify the pathophysiological cause to determine the most effective treatment.

For all the foregoing, we believe further research is needed to identify the pathophysiological basis of CME so that we can develop effective therapeutic algorithms for CME, regardless of the cause. For there may not be the adequate improvement unless the underlying cause is treated, and indeed, the CME could reappear. 


\section{References}

1. Irvine SR. A newly defined vitreous syndrome following cataract surgery: Interpreted according to recent concepts of the structure of the vitreous, the seventh francis I. proctor lecture. Am J Ophthalmol. 1953 May;36(5):599-619. 2. Cho H, Madu A. Etiology and treatment of the inflammatory causes of cystoid macular edema. J Inflamm Res. 2009 Oct;2:37-43.

3. Zur D, Fischer N, Tufail A, Mones J, Loewenstein A. Postsurgical cystoid macular edema. Eur J Ophthalmol. 2011 Aug;21 Suppl 6:S62-8.

4. Bergman M, Laatikainen L. Cystoid macular oedema after complicated cataract surgery and implantation of an anterior chamber lens. Acta 0phthalmol. 1994 Apr;72(2):178-80.

5. Johnson MW. Etiology and treatment of macular edema. Am J Ophthalmol. 2009 Jan;147(1):11-21. e1.

6. Bradford DJ, Wilkinson C, Bradford JR RH. Cystoid macular edema following extracapsular cataract extraction and posterior chamber intraocular lens implantation. Retina. 1988 Feb;8(3):161-4

7. Rotsos TG, Moschos MM. Cystoid macular edema. Clin Ophthalmol. 2008 Dec;2(4):919-30.

8. Sevim MS, Sanisoglu H, Turkyilmaz K. Intravitreal triamcinolone acetonide versus pars plana vitrectomy for pseudophakic cystoid macular edema. Cur Eye Res. 2012 Aug;37(12):1165-10.

9. Miyake K, Ota I, Miyake G, Numaga J. Nepafenac $0.1 \%$ versus fluorometholone $0.1 \%$ for preventing cystoid macular edema after cataract surgery. Cataract Refract Surg. 2011 Sep;37(9):1581-8.

10. Kessel L, Tendal B, Jørgensen KJ, Erngaard D, Flesner P, Andresen JL, et al. Post-cataract prevention of inflammation and macular edema by steroid and nonsteroidal anti-inflammatory eye drops: A systematic review. Ophthalmology. 2014 Oct;121(10):1915-24

11. Tranos PG, Tsaousis KT, Vakalis AN, Asteriades S, Pavesio CE. Long-term follow-up of inflammatory cystoid macular edema. Retina. 2012 Sep;32(8):1624-8. 12. Ouyang Y, Keane PA, Sadda SR, Walsh AC. Detection of cystoid macular edema with three-dimensional optical coherence tomography versus fluorescein angiography. Invest Ophthalmol Vis Sci. 2010 0ct;51(10):5213-8.

13. Brar M, Yuson R, Kozak I, Mojana F, Cheng L, Bartsch DU, et al. Correlation between morphologic features on spectral-domain optical coherence tomography and angiographic leakage patterns in macular edema. Retina. $2010 \mathrm{Mar} ; 30(3): 383-9$.

14. Pelosini L, Hull CC, Boyce JF, MCHugh D, Stanford MR, Marshall J. Optical coherence tomography may be used to predict visual acuity in patients with macular edema. Invest Ophthalmol Vis Sci. 2011 Apr;52(5):2741-8.

15. Fu A, Bui A, Roe R, Ahmed I, Ai E. Cystoid macular edema. In: Yanoff M DJ, ed. Ophtalmology. ; 2009:696-701.

16. Manousaridis K, Gupta R. Risperidone-related bilateral cystoid macular oedema. Garefe's Arch Clin Exp 0phtalmol. 2013 Mar;251(3):1037-8

17. Telander DG, Sarraf D. Cystoid macular edema with docetaxel chemotherapy and the fluid retention syndrome. Semin Ophthalmol. 2007 JulSep;22(3):151-3.

18. Joshi MM, Garretson BR. Paclitaxel maculopathy. Arch Ophthalmol. 2007 May; 125(5):709-10.

19. Ryan EH,Jr, Han DP, Ramsay RC, Cantrill HL, Bennett SR, Dev S, et al. Diabetic macular edema associated with glitazone use. Retina. 2006 MayJun;26(5):562-70.

20. Charalampidou S, Nolan J, Beatty S. The natural history of tractional cystoid macular edema. Retina. 2012 Nov-Dec;32(10):2045-51.

21. Jun JJ, Duker JS, Baumal CR, McCabe F, Reichel E, Rogers AH, et al. Cystoid macular edema without macular thickening: A retrospective optical coherence tomographic study. Retina. 2010 Jun;30(6):917-23.

22. Al Faran A, Mousa A, Al Shamsi H, Al Gaeed A, Chazi NG. Spectral domain optical coherence tomography predictors of visual outcome in diabetic cystoid macular edema after bevacizumab injection. Retina. 2014 Jun;34(6):1208-15. 23. Kim JT, Lee DH, Joe SG, Kim JG, Yoon YH. Changes in choroidal thickness in relation to the severity of retinopathy and macular edema in type 2 diabetic patients. Invest Ophthalmol Vis Sci. 2013 May;54(5):3378-84.

24. Ebrahimiadib N, Riazi-Esfahani M. Autofluorescence imaging for diagnosis and follow-up of cystoid macular edema. J Ophthalmic Vis Res. 2012 Jul; $7(3): 261-7$.

25. Flach AJ. Topical nonsteroidal antiinflammatory drugs in ophthalmology. Int Ophthalmol Clin. 2002 Winter;42(1):1-11.

26. Yilmaz T, Cordero-Coma M, Gallagher M. Ketorolac therapy for the prevention of acute pseudophakic cystoid macular edema: A systematic review. Eye. 2011 Feb;26(2):252-8.

27. Burnett J, Tessler H, Isenberg S, Tso MO. Double-masked trial of fenoprofen sodium: Treatment of chronic aphakic cystoid macular edema. Ophthalmic Surg. 1983 Feb;14(2):150-2.

28. Ramezani A, Fard Esmaeilpour N, Eskandari A, Rabbanikhah Z, Soheilian $R$, Soheilian M. Intravitreal diclofenac for refractory uveitic cystoid macular edema. J Ophthalmic Vis Res. 2013 Jan;8(1):47-52.

29. Shelsta HN, Jampol LM. Pharmacologic therapy of pseudophakic cystoid macular edema: 2010 update. Retina. 2011 Jan;31(1):4-12.

30. Abe T, Hayasaka S, Nagaki Y, Kadoi C, Matsumoto M, Hayasaka Y. Pseudophakic cystoid macular edema treated with high-dose intravenous methylprednisolone. J Cataract Refract Surg. 1999 Sep;25(9):1286-8.

31. Wakefield D, McCluskey P, Penny R. Intravenous pulse methylprednisolone therapy in severe inflammatory eye disease. Arch Ophthalmol. 1986 Jun; 104(6):847-51.

32. Hainsworth DP, Pearson PA, Conklin JD, Ashton P. Sustained release intravitreal dexamethasone. J Ocul Pharmacol Ther. 1996 Spring;12(1):57-63.

33. Jaffe GJ, Ben-nun J, Guo H, Dunn JP, Ashton P. Fluocinolone acetonide sustained drug delivery device to treat severe uveitis. Ophthalmology. 2000 Nov; $107(11): 2024-33$.

34. Koutsandrea C, Moschos MM, Brouzas D, Loukianou E, Apostolopoulos M, Moschos M. Intraocular triamcinolone acetonide for pseudophakic cystoid macular edema: Optical coherence tomography and multifocal electroretinography study. Retina. 2007 Feb;27(2):159-64.

35. Shen BY, Punjabi OS, Lowder CY, Sears JE, Singh RP. Early treatment response of fluocinolone (retisert) implantation in patients with uveitic macular edema: An optical coherence tomography study. Retina. 2013 Apr;33(4):873-7. 36. Jonas JB, Kreissig I, Söfker A, Degenring RF. Intravitreal injection of triamcinolone for diffuse diabetic macular edema. Arch Ophthalmol. 2003 Jan; 121(1):57-61.

37. Avitabile T, Longo A, Reibaldi A. Intravitreal triamcinolone compared with macular laser grid photocoagulation for the treatment of cystoid macular edema. Am J Ophthalmol. 2005 0ct;140(4):695-702.

38. Loewenstein A, Goldstein M. Intravitreal triamcinolone acetonide for diabetic macular edema. IMAJ. 2006 Jun;8(6):426.

39. Androudi S, Letko E, Meniconi M, Papadaki T, Ahmed M, Foster CS. Safety and efficacy of intravitreal triamcinolone acetonide for uveitic macular edema. Ocul Immunol Inflamm. 2005 Apr-Jun;13(2-3):205-12.

40. Jaffe CJ, Martin D, Callanan D, et al. Fluocinolone acetonide implant (retisert) for noninfectious posterior uveitis: Thirty-four-week results of a multicenter randomized clinical study. Ophthalmology. 2006 Jun;113(6):1020-7.

41. Coma MC, Sobrin L, Onal S, Christen W, Foster CS. Intravitreal bevacizumab for treatment of uveitic macular edema. Ophthalmology. 2007 Aug;114(8):1574-1579.

42. Jabs DA, Rosenbaum JT, Foster CS, Holland CN, Jaffe CJ, Louie JS, et al. Guidelines for the use of immunosuppressive drugs in patients with ocular inflammatory disorders: Recommendations of an expert panel. Am J Ophthalmol. 2000 Oct;130(4):492-513.

43. Kita M, Marmor MF. Effects on retinal adhesive force in vivo of metabolically active agents in the subretinal space. Invest Ophthalmol Vis Sci. 1992 May;33(6):1883-7.

44. Fishman CA, Gilbert LD, Fiscella RG, Kimura AE, Jampol LM. Acetazolamide 
for treatment of chronic macular edema in retinitis pigmentosa. Arch 0phthalmol. 1989 Oct;107(10):1445-52.

45. Cunningham ET Jr, Adamis AP, AltaweelM, Aiello LP, Bressler NM, D'Amico DJ, et al; Macugen Diabetic Retinopathy Study Group. A phase II randomized double-masked trial of pegaptanib, an Anti-Vascular endothelial growth factor aptamer, for diabetic macular edema. Ophthalmology. 2005 Oct;112(10):1747-57.

46. Mitropoulos PG, Chatziralli IP, Peponis VG, Drakos E, Parikakis EA. Intravitreal ranibizumab for the treatment of irvine-gass syndrome. Ocul Immunol Inflamm. 2015 Jun; 23(3):225-31

47. Lott MN, Schiffman JC, Davis JL. Bevacizumab in inflammatory eye disease. Am J Ophthalmol. 2009 Nov; 148(5):711-7.

48. Chasemi Falavarjani K, Parvaresh MM, Modarres M, Hashemi M, Samiy N. Intravitreal bevacizumab for pseudophakic cystoid macular edema; a systematic review. J Ophthalmic Vis Res. 2012 Jul; 7(3):235-9.

49. Moschos MM, Moschos M. Intraocular bevacizumab for macular ede ma due to CRVO. A multifocal-ERG and OCT study. Doc Ophthalmol. 2008 Mar;116(2):147-52.

50. Iturralde D, Spaide RF, Meyerle CB, Klancnik JM, Yannuzzi LA, Fisher YL, et al. Intravitreal bevacizumab (avastin) treatment of macular edema in central retinal vein occlusion: A short-term study. Retina. $2006 \mathrm{Mar} ; 26(3): 279-84$.

51. Central Vein Occlusion Study Group. Evaluation of grid pattern photocoagulation for macular edema in central vein occlusion: The central vein occlusion study group M report. Ophthalmology. 1995 Oct;102(10):1425-33.

52. Branch Vein Occlusion Study Group. Argon laser photocoagulation for macular edema in branch vein occlusion. Am J Ophthalmol. 1984 Sep;98(3):271-82. 53. Early Treatment Diabetic Retinopathy Study Research Group. Techniques for scatter and local photocoagulation treatment of diabetic retinopathy: Early treatment diabetic retinopathy study report no. 3. Int Ophthalmol Clin. 1987 Winter;27(4):254-64.

54. Harbour JW, Smiddy WE, Rubsamen PE, Murray TC, Davis JL, Flynn HW. Pars plana vitrectomy for chronic pseudophakic cystoid macular edema. Am J Ophthalmol. 1995 Sep;120(3):302-7.

55. Nasrallah FP, Jalkh AE, Van Coppenolle F, et al. The role of the vitreous in diabetic macular edema. Ophthalmology. 1988 0ct;95(10):1335-9.

56. Packer M, Lowe J, Fine H. Incidence of acute postoperative cystoid macular edema in clinical practice. J Cataract Refract Surg. 2012 Dec;38(12):2108-11.

\section{Acknowledgments}

The authors would like to thank Alistair Ross for correcting the English version of this work.

\section{Conflict of Interest Statement at Funding}

The author has no funding, financial relationships or conflicts of interest to disclose.

Author Contributions

Conception and design the work/idea, Analysis and interpretation of data, Approval of the final version: JMR, NBA, JZO, ESG, JJS. Collect data/obtaining results, Write the manuscript: NBA, JZO, ESG. Critical revision of the manuscript: JMR. Administrative or technical advice: JJS

Cite as:

Bravo-Alcobendas N, Zulueta J, Salobrar-García E, Salazar JJ, Ramírez JM. Cystoid Macular Edema: Causes, Diagnosis and Treatment. Int J Med Students. 2015 Sep-Dec;3(3):131-9. 